\title{
There are no Phantom Pairs of Mappings to 1-Dimensional CW-Complexes
}

by

\author{
Sibe MARDEŠIĆ
}

Presented by Czestaw BESSAGA

Summary. Two mappings from a CW-complex to a 1-dimensional CW-complex are homotopic if and only if their restrictions to finite subcomplexes are homotopic.

1. Introduction. Let $f, g: P \rightarrow P^{\prime}$ be two mappings between $\mathrm{CW}$ complexes. Clearly, if $f$ and $g$ are homotopic, $f \simeq g$, then for every finite subcomplex $Q \subseteq P$, the restrictions $f|Q, g| Q$ are also homotopic. In this paper we will prove that the converse implication holds provided $P^{\prime}$ has dimension $\leq 1$, i.e., we will prove the following theorem.

Theorem 1. Let $P, P^{\prime}$ be $C W$-complexes and let $f, g: P \rightarrow P^{\prime}$ be mappings such that, for every finite subcomplex $Q \subseteq P$, the restrictions $f|Q, g| Q$ are homotopic. If $\operatorname{dim} P^{\prime} \leq 1$, then $f \simeq g$.

In homotopy theory a mapping $f: P \rightarrow Y$ from a $\mathrm{CW}$-complex $P$ to a topological space $Y$ is called an essential phantom mapping of the second kind provided $f$ is essential, i.e., it is not homotopic to a constant mapping, but its restriction to any finite subcomplex $Q$ of $P$ is homotopic to a constant mapping [5]. A generalization of this notion is the notion of an essential phantom pair of mappings of the second kind. This is a pair of nonhomotopic mappings $f, g: P \rightarrow Y$ whose restrictions $f|Q, g| Q$ to every finite subcomplex $Q$ of $P$ are homotopic. Consequently, Theorem 1 can be restated as follows.

THEOREM 1'. There are no essential phantom pairs of mappings of the second kind from a $C W$-complex $P$ to a $C W$-complex $P^{\prime}$ with $\operatorname{dim} P^{\prime} \leq 1$.

2000 Mathematics Subject Classification: 57M05, 55S37, $20 \mathrm{E} 05$.

Key words and phrases: homotopy classification of mappings, 1-dimensional CWcomplexes, phantom pairs of mappings, fundamental group, free group. 
REMARK 1. In Theorem 1 the assumption $f|Q \simeq g| Q$ for finite subcomplexes $Q \subseteq P$ cannot be replaced by the assumption that the restrictions of $f$ and $g$ to 1-cells of $P$ be homotopic (see Example 2 in Section 4).

2. Equivalent forms of Theorem 1. Theorem 1 is equivalent to the following theorem (the author owes this remark to J. Dydak).

Theorem 2. Let $P, P^{\prime}$ be connected $C W$-complexes and let $f, g: P \rightarrow P^{\prime}$ be mappings such that, for every finite subcomplex $Q \subseteq P$, the restrictions $f|Q, g| Q$ are homotopic. If $\operatorname{dim} P=\operatorname{dim} P^{\prime}=1$, then $f \simeq g$.

Obviously, Theorem 1 implies Theorem 2 . To prove the converse, consider $f, g: P \rightarrow P^{\prime}$ such that $f|Q \simeq g| Q$ for all finite subcomplexes $Q$ of $P$. Since every point $u \in P$ belongs to a finite subcomplex $Q$ of $P$, the points $f(u), g(u)$ belong to the same component of $P^{\prime}$. Hence, $f$ and $g$ map a component of $P$ to the same component of $P^{\prime}$. Therefore, it suffices to prove Theorem 1 under the additional assumption that $P$ and $P^{\prime}$ are connected.

If $\operatorname{dim} P^{\prime}=0$, then $P^{\prime}$ is a point $*^{\prime}$, and thus $f=g$. Therefore, it suffices to consider the case when $\operatorname{dim} P^{\prime}=1$. If $\operatorname{dim} P=0$, then $P$ is a point $*$, and thus $f=f|* \simeq g| *=g$. Therefore, we can assume that $\operatorname{dim} P \geq 1$. Consider the 1 -skeleton $P^{1}$ of $P$. Clearly, the restrictions $f\left|P^{1}, g\right| P^{1}: P^{1} \rightarrow P^{\prime}$ satisfy the assumptions of Theorem 2, and therefore there exists a homotopy $h^{1}$ : $P^{1} \times I \rightarrow P^{\prime}$ which connects $f \mid P^{1}$ and $g \mid P^{1}$.

It is well known that every connected $\mathrm{CW}$-complex of dimension 1 is an Eilenberg-Mac Lane complex of type $K(G, 1)$ (see, e.g., [1, Example 1B.1]). Therefore, the homotopy groups $\pi_{n}\left(P^{\prime}\right)$ are zero for $n \geq 2$. Consequently, there are no obstructions to extending a homotopy $h^{n-1}: P^{n-1} \times I \rightarrow P^{\prime}$ of the $(n-1)$-skeleton $P^{n-1}$ of $P$ to a homotopy $h^{n}: P^{n} \times I \rightarrow P^{\prime}$ of its $n$-skeleton $P^{n}$ (use, e.g., Lemma 4.7 of [1]). Proceeding in this way one obtains a homotopy connecting $f$ to $g$.

We will now prove that Theorem 2 is equivalent to the following theorem.

Theorem 3. Let $P, P^{\prime}$ be connected 1-dimensional $C W$-complexes having only one 0 -cell $*$ and $*^{\prime}$, respectively and let $f, g: P \rightarrow P^{\prime}$. If for every finite subcomplex $Q \subseteq P$ the restrictions $f|Q, g| Q$ are homotopic, then $f \simeq g$.

Obviously, Theorem 2 implies Theorem 3. To prove the converse, consider $f, g: P \rightarrow P^{\prime}$ such that $f|Q \simeq g| Q$ for all finite subcomplexes $Q$ of $P$. It is well known that every connected 1-dimensional $\mathrm{CW}$-complex contains a maximal tree (see, e.g., [1, Proposition 1A.1]). Let $T$ and $T^{\prime}$ be maximal trees in $P$ and $P^{\prime}$, respectively. Since the pair $(P, T)$ has the homotopy extension property and $T$ is contractible, the quotient mapping $q: P \rightarrow P / T$ is a homotopy equivalence, and thus admits a homotopy inverse $r: P / T \rightarrow P$ (see, e.g., $\left[1\right.$, Proposition 0.17]). Analogously, the quotient mapping $q^{\prime}: P^{\prime} \rightarrow P^{\prime} / T^{\prime}$ admits a homotopy inverse $r^{\prime}: P^{\prime} / T^{\prime} \rightarrow P^{\prime}$. If $P^{\prime}=T^{\prime}$, then $P^{\prime}$ is contractible, 
and thus $f \simeq g$. Otherwise, $R^{\prime}=P^{\prime} / T^{\prime}$ is a connected 1-dimensional $\mathrm{CW}$ complex having the point $*^{\prime}=T^{\prime}$ as its only 0-cell. Analogously, if $P=T$, then $P$ contracts to a point $u_{0} \in P$, which is a 0 -cell of $P$. Consequently, $f$ is homotopic to the constant $f\left(u_{0}\right)$ and $g$ is homotopic to the constant $g\left(u_{0}\right)$. But $f\left|u_{0} \simeq g\right| u_{0}$, and thus $f \simeq g$. Otherwise, $R=P / T$ is a connected 1-dimensional CW-complex having the point $*=T$ as its only 0 -cell. To complete the proof it suffices to consider the case when $P=T$ and $P^{\prime}=T^{\prime}$. Let $f^{\prime}, g^{\prime}: R \rightarrow R^{\prime}$ be defined by $f^{\prime}=q^{\prime} f r$ and $g^{\prime}=q^{\prime} g r$. If $S \subseteq R$ is a finite subcomplex of $R$, then $S$ and $r(S)$ are compact. Therefore, $r(S)$ is contained in a finite subcomplex $Q$ of $P$. By assumption, $f|Q \simeq g| Q$ and thus also $f r|S \simeq g r| S$. It follows that $f^{\prime}\left|S=q^{\prime} f r\right| S \simeq q^{\prime} g r\left|S=g^{\prime}\right| S$, i.e., the restrictions of $f^{\prime}$ and $g^{\prime}$ to all finite subcomplexes of $R$ are homotopic. Now Theorem 3 shows that $f^{\prime} \simeq g^{\prime}$, i.e., $q^{\prime} f r \simeq q^{\prime} g r$. Since $q^{\prime}$ and $r$ are homotopy equivalences, it follows that also $f \simeq g$.

3. A theorem on free groups. To prove Theorem 3 we need the following theorem on free groups.

TheOREM 4. Let $F$ be a free group and let $\left(\alpha_{i}\right),\left(\beta_{i}\right), i \in M$, be two collections of elements from $F$. If for every finite subset $L \subseteq M$, there exists an element $\gamma_{L} \in F$ such that $\alpha_{i}=\gamma_{L} \beta_{i} \gamma_{L}^{-1}$ for every $i \in L$, then there exists an element $\gamma \in F$ such that $\alpha_{i}=\gamma \beta_{i} \gamma^{-1}$ for every $i \in M$.

In the proof of Theorem 4 we will use some well-known facts concerning free groups. They are stated in the following proposition.

Proposition 1. In a free group $F$ the following statements hold:

(i) If $a \in F, a \neq 1, n \in \mathbb{Z}$ and $a^{n}=1$, then $n=0$.

(ii) If $a, b \in F$ and $m, n \in \mathbb{Z}$ are integers different from 0 such that $a^{m}$ and $b^{n}$ commute, then there exist an element $c \in F$ and integers $r, s \in \mathbb{Z}$ such that $a=c^{r}, b=c^{s}$.

(iii) If $a, b \in F, a \neq 1, b \neq 1$ and $n \in \mathbb{Z}, n \neq 0$, then $a^{n}=b^{n}$ implies $a=b$.

(iv) If $a, b, c \in F$ are different from 1 , if $a$ and $b$ commute and if $b$ and c commute, then also $a$ and c commute.

For a proof of (i) see [3, Corollary 1.2.2] or [2, Proposition 2.16]. For (ii) see $\left[3,1.4\right.$, Problems 4 and 6] or [2, Proposition 2.17]. For (iii) note that $a^{n}$ and $b^{n}$ commute, and therefore, by (ii), there exist $c \in F$ and $r, s \in \mathbb{Z}$ such that $a=c^{r}$ and $b=c^{s}$. Consequently, $c^{n r}=c^{n s}$, and thus $c^{n r-n s}=1$. Since $c \neq 1$, (i) implies that $n r-n s=0$, and thus $r=s$, which yields the desired conclusion that $a=b$. For (iv) see [2, Proposition 2.18].

Proof of Theorem 4. For an arbitrary $i \in M$, consider the singleton $\{i\}$ and put $\gamma_{i}=\gamma_{\{i\}}$. Note that $\alpha_{i}=\gamma_{i} \beta_{i} \gamma_{i}^{-1}$. If for a given $i \in M, \beta_{i}=1$, then 
$\alpha_{i}=1$, and thus $\alpha_{i}=\gamma \beta_{i} \gamma^{-1}$ for any $\gamma \in F$. Therefore, there is no loss of generality in assuming that $\beta_{i} \neq 1$ for all $i \in M$. Denote by $B$ the subgroup of $F$ generated by all $\beta_{i}, i \in M$. Being a subgroup of a free group, $B$ is also a free group (see [3, Corollary 2.9] or [2, Proposition 3.3]). We distinguish two cases: I, when $B$ is commutative, and II, when $B$ is not commutative.

In case I, we fix an arbitrary $k \in M$. We will show that $\gamma=\gamma_{k}$ is as required, i.e., $\alpha_{i}=\gamma_{k} \beta_{i} \gamma_{k}^{-1}$ for all $i \in M$. Indeed, since the only commutative group which is free is the free cyclic group (see [3, 2.4, Problem 2] or [2, Proposition 3.1]), $B$ is cyclic. Let $\{\beta\}$ be a basis of $B$. Then every element $\beta_{i}, i \in M$, is of the form $\beta_{i}=\beta^{r_{i}}$, where $r_{i} \in \mathbb{Z}$. By the assumptions of Theorem 4, for an arbitrary $i \in M$ and the finite set $L=\{i, k\} \subseteq M$, there exists $\gamma_{i k}=\gamma_{\{i, k\}} \in F$ such that $\alpha_{i}=\gamma_{i k} \beta_{i} \gamma_{i k}^{-1}$ and $\alpha_{k}=\gamma_{i k} \beta_{k} \gamma_{i k}^{-1}$. Since $\beta_{i}=\beta^{r_{i}}$, we see that

$$
\alpha_{i}=\gamma_{i} \beta^{r_{i}} \gamma_{i}^{-1}=\left(\gamma_{i} \beta \gamma_{i}^{-1}\right)^{r_{i}} \quad \text { and } \quad \alpha_{i}=\gamma_{i k} \beta^{r_{i}} \gamma_{i k}^{-1}=\left(\gamma_{i k} \beta \gamma_{i k}^{-1}\right)^{r_{i}} \text {. }
$$

Therefore, $\left(\gamma_{i} \beta \gamma_{i}^{-1}\right)^{r_{i}}=\left(\gamma_{i k} \beta \gamma_{i k}^{-1}\right)^{r_{i}}$. Note that $r_{i} \neq 0$, because $r_{i}=0$ would imply $\beta_{i}=1$. Moreover, $\gamma_{i} \beta \gamma_{i}^{-1} \neq 1$, because $\gamma_{i} \beta \gamma_{i}^{-1}=1$ would imply $\beta=1$ hence, also $\beta_{i}=1$. Similarly, $\gamma_{i k} \beta \gamma_{i k}^{-1} \neq 1$. By Proposition 1(iii), one concludes that $\gamma_{i} \beta \gamma_{i}^{-1}=\gamma_{i k} \beta \gamma_{i k}^{-1}$. An analogous argument shows that $\gamma_{k} \beta \gamma_{k}^{-1}=\gamma_{i k} \beta \gamma_{i k}^{-1}$. Consequently, $\gamma_{i} \beta \gamma_{i}^{-1}=\gamma_{k} \beta \gamma_{k}^{-1}$, and thus $\left(\gamma_{i} \beta \gamma_{i}^{-1}\right)^{r_{i}}=$ $\left(\gamma_{k} \beta \gamma_{k}^{-1}\right)^{r_{i}}$. Since $\alpha_{i}=\left(\gamma_{i} \beta \gamma_{i}^{-1}\right)^{r_{i}}$ and $\gamma_{k} \beta_{i} \gamma_{k}^{-1}=\left(\gamma_{k} \beta \gamma_{k}^{-1}\right)^{r_{i}}$, we obtain the desired relation $\alpha_{i}=\gamma_{k} \beta_{i} \gamma_{k}^{-1}$ for all $i \in M$.

In case II, $B$ is not commutative, so there exist $k, l \in M$ such that $\beta_{k}$ and $\beta_{l}$ do not commute. Consider the fixed subset $\{k, l\} \subseteq M$ and put $\gamma_{k l}=\gamma_{\{k, l\}}$. For an arbitrary $i \in M$, consider the subset $\{i, k, l\} \subseteq M$ and put $\gamma_{i k l}=\gamma_{\{i, k, l\}}$. Let us show that

$$
\gamma_{i k l}=\gamma_{k l} \quad \text { for all } i \in M \text {. }
$$

Indeed, since $k \in\{k, l\} \cap\{i, k, l\}$, we see that $\alpha_{k}=\gamma_{k l} \beta_{k} \gamma_{k l}^{-1}$ and $\alpha_{k}=$ $\gamma_{i k l} \beta_{k} \gamma_{i k l}^{-1}$. Consequently,

$$
\gamma_{k l} \beta_{k} \gamma_{k l}^{-1}=\gamma_{i k l} \beta_{k} \gamma_{i k l}^{-1}
$$

This shows that $\gamma_{k l}^{-1} \gamma_{i k l}$ commutes with $\beta_{k}$. Analogously, $\gamma_{k l}^{-1} \gamma_{i k l}$ commutes with $\beta_{l}$. Recall that $\beta_{k} \neq 1$ and $\beta_{l} \neq 1$. Therefore, if one would also have $\gamma_{k l}^{-1} \gamma_{i k l} \neq 1$, Proposition 1(iv) would imply that $\beta_{k}$ commutes with $\beta_{l}$, which is not the case. We have thus proved that $\gamma_{k l}^{-1} \gamma_{i k l}=1$, i.e., $\gamma_{k l}=\gamma_{i k l}$, as desired.

Since $\alpha_{i}=\gamma_{i k l} \beta_{i} \gamma_{i k l}^{-1}$ for all $i \in M$, the equality $\gamma_{i k l}=\gamma_{k l}$ implies that $\alpha_{i}=\gamma_{k l} \beta_{i} \gamma_{k l}^{-1}$ for all $i \in M$. Consequently, $\gamma=\gamma_{k l}$ is as required.

EXAMPLE 1. Let $F$ be the free group of rank 2 with basis $\left\{\beta_{1}, \beta_{2}\right\}$. Let $\alpha_{1}=\beta_{2}^{-1} \beta_{1} \beta_{2}$ and $\alpha_{2}=\beta_{1}^{-1} \beta_{2} \beta_{1}$. Then there is no $\gamma \in F$ such that $\alpha_{i}=$ $\gamma \beta_{i} \gamma^{-1}$ for $i=1,2$. 
To verify the assertion, assume that $\gamma \in F$ is such that $\alpha_{i}=\gamma \beta_{i} \gamma^{-1}$ for $i=1,2$, i.e.,

$$
\beta_{2}^{-1} \beta_{1} \beta_{2}=\gamma \beta_{1} \gamma^{-1} \text { and } \beta_{1}^{-1} \beta_{2} \beta_{1}=\gamma \beta_{2} \gamma^{-1} \text {. }
$$

The first of these relations shows that $\beta_{2} \gamma$ commutes with $\beta_{1}$. By Proposition 1(ii), there exist $\xi \in F$ and $r, s \in \mathbb{Z}$ such that $\beta_{1}=\xi^{r}$ and $\beta_{2} \gamma=\xi^{s}$. There is no loss of generality in assuming that $r \geq 0$ (if not, replace $\xi$ by $\xi^{-1}$ ). Since $\beta_{1}$ belongs to a basis of $F$, one cannot have $r \geq 2$, and thus $r=1$. Consequently, $\beta_{2} \gamma=\left(\beta_{1}\right)^{s}$. Analogously, there exists an integer $s^{\prime}$ such that $\beta_{1} \gamma=\left(\beta_{2}\right)^{s^{\prime}}$. It follows that

$$
\beta_{2}^{-1}\left(\beta_{1}\right)^{s}=\gamma=\beta_{1}^{-1}\left(\beta_{2}\right)^{s^{\prime}} .
$$

However, this is impossible because $\beta_{2}^{-1}\left(\beta_{1}\right)^{s}$ and $\beta_{1}^{-1}\left(\beta_{2}\right)^{s^{\prime}}$ are reduced words, beginning with $\beta_{2}^{-1}$ and $\beta_{1}^{-1}$, respectively. Therefore, they cannot represent the same element of $F$.

4. Proof of Theorem 3. To prove Theorem 3, we will use Theorem 4 and some elementary facts concerning the homotopy of loops in a pointed space $\left(Y, *^{\prime}\right)$. In particular, denote by $\left(S^{1}, *\right)$ the standard 1-sphere $\{z \in \mathbb{C}$ : $|z|=1\}$ with the basepoint $*=1$ and let $e: I \rightarrow S^{1}$ be the exponential mapping, $e(t)=e^{2 \pi i t}, t \in I=[0,1]$. By a loop $a$ in $Y$, based at $*^{\prime}$, we mean a mapping $a: S^{1} \rightarrow Y$ such that $a(*)=*^{\prime}$. Note that $a$ determines the path $\widetilde{a}=a e: I \rightarrow Y$, which has the property that $\widetilde{a}(0)=\widetilde{a}(1)=*^{\prime}$. Conversely, every path $\widetilde{a}: I \rightarrow S^{1}$ having the latter property determines a unique loop $a$ such that $\widetilde{a}=a e$. The composition of two loops $a_{1}, a_{2}: S^{1} \rightarrow Y$, based at $*^{\prime}$, is the only loop $a_{1} a_{2}$ such that $\left(a_{1} a_{2}\right) e=\widetilde{a}_{1} \widetilde{a}_{2}$. We will say that the loops $a, b$, based at $*^{\prime}$, are (freely) homotopic provided there exists a homotopy $H: S^{1} \times I \rightarrow Y$ such that $H(u, 0)=a(u)$ and $H(u, 1)=b(u)$ for $u \in S^{1}$. Note that the formula $\widetilde{c}(t)=H(*, t)$ determines a path $\widetilde{c}: I \rightarrow Y$ such that $\widetilde{c}(0)=H(*, 0)=a(*)=*^{\prime}$ and $\widetilde{c}(1)=H(*, 1)=b(*)=*^{\prime}$. Therefore, $\widetilde{c}$ determines a loop $c: S^{1} \rightarrow Y$ based at $*^{\prime}$. We will say that $H$ is a $c$-homotopy and the loops $a$ and $b$ are $c$-homotopic. Let $\alpha, \beta, \gamma \in \pi_{1}\left(Y, *^{\prime}\right)$ be the homotopy classes of the loops $a, b, c$. Then the following elementary lemma holds (see [4, Theorem II.8.2]).

LEMMA 1. Let $a, b, c$ be loops in a pointed space $\left(Y, *^{\prime}\right)$ and let $\alpha, \beta, \gamma$ be the corresponding classes in $\pi_{1}\left(Y, *^{\prime}\right)$. Then the following two conditions are equivalent:

(i) the loops $a$ and $b$ are c-homotopic;

(ii) $\alpha=\gamma \beta \gamma^{-1}$.

Proof. If (i) holds, then there is a $c$-homotopy $H: S^{1} \times I \rightarrow Y$ which connects $a$ and $b$ and $H(*, t)=\widetilde{c}(t)$ for $t \in I$. Therefore, $\widetilde{H}: I \times I \rightarrow Y$ given by $\widetilde{H}=H(e \times 1)$ is a homotopy which connects $\widetilde{a}$ to $\widetilde{b}$. Moreover, $\widetilde{H}(0, t)=$ 
$H(e(0), t)=H(*, t)=\widetilde{c}(t)$ and $\widetilde{H}(1, t)=H(e(1), t)=H(*, t)=\widetilde{c}(t)$. Clearly, $\widetilde{H}$ gives rise to a homotopy $\widetilde{G}: I \times I \rightarrow Y$ which connects the loops $\widetilde{a}$ and $\widetilde{c} \widetilde{b} \widetilde{c}^{-1}$ and is fixed at the two end-points 0 , 1, i.e., it is a homotopy rel $\partial I$. Now $\widetilde{G}$ determines a homotopy $G: S^{1} \times I \rightarrow Y$ such that $\widetilde{G}=G(e \times 1)$. Note that $G$ connects the loops $a$ and $c b c^{-1}$ and is fixed at the basepoint *, i.e., it is a homotopy rel $*$. Indeed, if $s \in I$ and $u=e(s)$, then

$$
G(u, 0)=G(e(s), 0)=\widetilde{G}(s, 0)=\widetilde{a}(s)=a e(s)=a(u) .
$$

Similarly,

$$
G(u, 1)=G(e(s), 1)=\widetilde{G}(s, 1)=\widetilde{c} \widetilde{b} \widetilde{c}^{-1}(s)=c b c^{-1}(e(s))=c b c^{-1}(u) .
$$

Moreover, $G(*, t)=G(e(0), t)=\widetilde{G}(0, t)=*^{\prime}$. It follows that $\alpha=\gamma \beta \gamma^{-1}$, as required by (ii).

To prove (ii) $\Rightarrow($ i), it suffices to follow the steps of the above proof in the opposite order.

Proof of Theorem 3. First note that every mapping $f: P \rightarrow P^{\prime}$ is homotopic to a mapping $f^{\prime}: P \rightarrow P^{\prime}$ such that $f^{\prime}(*)=*^{\prime}$. Indeed, since $P^{\prime}$ is pathwise connected, there is a path $\omega: I \rightarrow P^{\prime}$ such that $\omega(0)=f(*)$ and $\omega(1)=*^{\prime}$. By the homotopy extension property for the pair $(P, *)$, there is a homotopy $H: P \times I \rightarrow P^{\prime}$ such that $H(u, 0)=f(u)$ for $u \in P$ and $H(*, s)=\omega(s)$ for $s \in I$. Define $f^{\prime}: P \rightarrow P^{\prime}$ by putting $f^{\prime}(u)=H(u, 1)$. Clearly, $f \simeq f^{\prime}$ implies $f^{\prime}|Q \simeq f| Q$ for every finite subcomplex $Q \subseteq P$. Moreover, $f^{\prime}(*)=H(*, 1)=\omega(1)=*^{\prime}$. Repeating the argument for $g$, we see that there is no loss of generality in assuming that $f$ and $g$ preserve the basepoints, i.e., $f(*)=g(*)=*^{\prime}$.

Being a connected 1-dimensional $\mathrm{CW}$-complex with a single 0 -cell $*, P$ is the wedge $\bigvee_{i \in M} P_{i}$ of a collection of copies $\left(P_{i}, *_{i}\right)$ of $\left(S^{1}, *\right), i \in M$. It is obtained from the coproduct $\bigsqcup_{i \in M} P_{i}$ by identifying all the basepoints $*_{i} \in$ $P_{i}$ to a single basepoint $*$ of $P$. Let $e_{i}: I \rightarrow P_{i}, i \in M$, denote the exponential mappings. It is well known that $\pi_{1}(P, *)$ is a free group, having as a basis the collection $\left[e_{i}\right], i \in M$, of homotopy classes (rel $\partial I$ ) of the loops $e_{i}$ (see, e.g., [1, Proposition 1A.2]). Analogous assertions hold for $P^{\prime}=\bigvee_{i \in M^{\prime}} P_{i}^{\prime}$.

For every $i \in M$, consider the loops $a_{i}=f \mid P_{i}$ and $b_{i}=g \mid P_{i}$ in $P^{\prime}$, based at $*^{\prime}$. By the assumptions of Theorem 3, $f|Q \simeq g| Q$ for every finite subcomplex $Q$ of $P$. In particular, this holds for $Q=P_{L}=\bigvee_{i \in L} P_{i}$ for any finite subset $L \subseteq M$. Therefore, there exists a homotopy $H_{L}: P_{L} \times I \rightarrow P^{\prime}$ which connects $f \mid Q$ and $g \mid Q$. Let $\widetilde{c}_{L}: I \rightarrow P^{\prime}$ be given by $\widetilde{c}_{L}(t)=H(*, t)$. Clearly, $H_{L} \mid P_{i} \times I$ is a $c_{L}$-homotopy connecting $a_{i}$ to $b_{i}$. Therefore, the implication (i) $\Rightarrow$ (ii) in Lemma 1 shows that the homotopy classes $\alpha_{i}=$ $\left[a_{i}\right], \beta_{i}=\left[b_{i}\right], \gamma_{L}=\left[c_{L}\right] \in \pi_{1}\left(P^{\prime}, *^{\prime}\right)$ satisfy $\alpha_{i}=\gamma_{L} \beta_{i} \gamma_{L}^{-1}$ for all $i \in L$. Now 
Theorem 4 shows that there exists $\gamma \in \pi_{1}\left(P^{\prime}, *^{\prime}\right)$ such that $\alpha_{i}=\gamma \beta_{i} \gamma^{-1}$ for all $i \in M$. Let $c$ be a representative of the class $\gamma$. Using the implication (ii) $\Rightarrow(\mathrm{i})$ of Lemma 1 , we conclude that, for every $i \in M$, there is a $c$-homotopy $H_{i}: P_{i} \times I \rightarrow P^{\prime}$, which connects the loops $a_{i}$ and $b_{i}$. Since $H_{i}(*, t)=\widetilde{c}(t)$ does not depend on $i$, the homotopies $H_{i}, i \in M$, extend to a well-defined homotopy $H: P \times I \rightarrow P^{\prime}$ which connects $f$ and $g$, because $H\left|P_{i} \times 0=H_{i}\right| P_{i} \times 0=a_{i}=f \mid P_{i}$ and $H\left|P_{i} \times 1=H_{i}\right| P_{i} \times 1=b_{i}=g \mid P_{i}$.

EXAmple 2. Let $P=P_{1} \vee P_{2}$ be the wedge of two copies of $S^{1}$. Let $\alpha_{i}, \beta_{i} \in \pi_{1}(P, *), i=1,2$, be as in Example 1 . Let $a_{i}, b_{i}$ be loops in $P$, based at $*$, such that $\alpha_{i}=\left[a_{i}\right], \beta_{i}=\left[b_{i}\right], i=1,2$, and let $f, g: P \rightarrow P$ be defined by $f\left|P_{i}=a_{i}, g\right| P_{i}=b_{i}$ for $i=1,2$. Then $f\left|P_{i} \simeq g\right| P_{i}$ for $i \in M$, but $f \not g$.

Consider the loops $c_{1}=b_{2}^{-1}$ and $c_{2}=b_{1}^{-1}$ and the corresponding classes $\gamma_{1}=\left[c_{1}\right]$ and $\gamma_{2}=\left[c_{2}\right]$. Since $\alpha_{1}=\gamma_{1} \beta_{1} \gamma_{1}^{-1}$ and $\alpha_{2}=\gamma_{2} \beta_{2} \gamma_{2}^{-1}$, Lemma 1 shows that $f\left|P_{i} \simeq g\right| P_{i}$ for $i \in M$. Now assume that $f \simeq g$. More precisely, let $g$ be $c$-homotopic to $f$, where $c: I \rightarrow P$ is a loop based at $*$. If $\gamma$ denotes the class of $c$, Lemma 1 shows that $\alpha_{i}=\gamma \beta_{i} \gamma^{-1}$ for $i=1,2$, which contradicts the assertions of Example 1.

Acknowledgements. The author wishes to express his gratitude to the referee of this paper for having improved the presentation and having suggested considerable simplifications in the proofs.

\section{References}

[1] A. Hatcher, Algebraic Topology, Cambridge Univ. Press, Cambridge, 2002.

[2] R. C. Lyndon and P. E. Schupp, Combinatorial Group Theory, Springer, Berlin, 1977.

[3] W. Magnus, A. Karrass and D. Solitar, Combinatorial Group Theory: Presentation of Groups in Terms of Generators and Relations, Interscience, New York, 1966.

[4] W. S. Massey, A Basic Course in Algebraic Topology, Grad. Texts in Math. 127, Springer, New York, 1991.

[5] C. A. McGibbon, Phantom maps, in: Handbook of Algebraic Topology, Chapter 25, I. M. James (ed.), Elsevier, Amsterdam, 1995, 1209-1257.

Sibe Mardešić

Department of Mathematics

University of Zagreb

Bijenička cesta 30

10002 Zagreb, P.O. Box 335, Croatia

E-mail: smardes@math.hr 